Louisiana State University

LSU Digital Commons

Faculty Publications

Department of Physics \& Astronomy

2-13-2017

\title{
Distributed angular double-slit interference with pseudo-thermal light
}

Lu Gao

School of Science

Seyed Mohammad Hashemi Rafsanjani

University of Rochester Institute of Optics

Yiyu Zhou

University of Rochester Institute of Optics

Zhe Yang

University of Rochester Institute of Optics

Omar S. Magaña-Loaiza

University of Rochester Institute of Optics

See next page for additional authors

Follow this and additional works at: https://digitalcommons.Isu.edu/physics_astronomy_pubs

\section{Recommended Citation}

Gao, L., Hashemi Rafsanjani, S., Zhou, Y., Yang, Z., Magaña-Loaiza, O., Mirhosseini, M., Zhao, J., Gao, B., \& Boyd, R. (2017). Distributed angular double-slit interference with pseudo-thermal light. Applied Physics Letters, 110 (7) https://doi.org/10.1063/1.4976575

This Article is brought to you for free and open access by the Department of Physics \& Astronomy at LSU Digital Commons. It has been accepted for inclusion in Faculty Publications by an authorized administrator of LSU Digital Commons. For more information, please contact ir@lsu.edu. 


\section{Authors}

Lu Gao, Seyed Mohammad Hashemi Rafsanjani, Yiyu Zhou, Zhe Yang, Omar S. Magaña-Loaiza, Mohammad Mirhosseini, Jiapeng Zhao, Boshen Gao, and Robert W. Boyd 


\section{Distributed angular double-slit interference with pseudo-thermal light}

Cite as: Appl. Phys. Lett. 110, 071107 (2017); https://doi.org/10.1063/1.4976575

Submitted: 06 January 2017 . Accepted: 02 February 2017 . Published Online: 14 February 2017

Lu Gao (D), Seyed Mohammad Hashemi Rafsanjani, Yiyu Zhou, Zhe Yang, Omar S. Magaña-Loaiza, Mohammad Mirhosseini, Jiapeng Zhao, Boshen Gao, and Robert W. Boyd
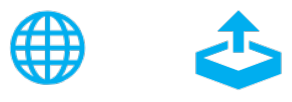

\section{ARTICLES YOU MAY BE INTERESTED IN}

Electrically driven and electrically tunable quantum light sources

Applied Physics Letters 110, 071102 (2017); https://doi.org/10.1063/1.4976197

Electrically tunable all-dielectric optical metasurfaces based on liquid crystals

Applied Physics Letters 110, 071109 (2017); https://doi.org/10.1063/1.4976504

Precision phase estimation based on weak-value amplification

Applied Physics Letters 110, 071105 (2017); https://doi.org/10.1063/1.4976312

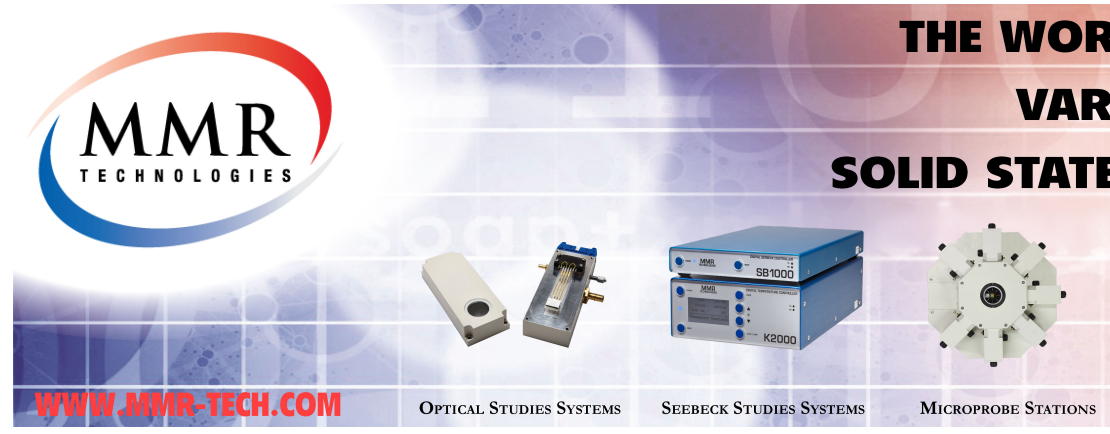

THE WORLD'S RESOURCE FOR VARIABLE TEMPERATURE 


\title{
Distributed angular double-slit interference with pseudo-thermal light
}

\author{
Lu Gao, , ,2,a) Seyed Mohammad Hashemi Rafsanjani, ${ }^{2}$ Yiyu Zhou, ${ }^{2}$ Zhe Yang, ${ }^{2,3}$ \\ Omar S. Magaña-Loaiza, ${ }^{2}$ Mohammad Mirhosseini, ${ }^{2}$ Jiapeng Zhao, ${ }^{2}$ Boshen Gao, ${ }^{2}$ \\ and Robert W. Boyd ${ }^{2,4}$ \\ ${ }^{1}$ School of Science, China University of Geosciences, Beijing 100083, China \\ ${ }^{2}$ The Institute of Optics, University of Rochester, Rochester, New York 14627, USA \\ ${ }^{3}$ State Key Laboratory of Low-dimensional Quantum Physics and Department of Physics, Tsinghua University, \\ Beijing 100084, China \\ ${ }^{4}$ Department of Physics and Max Planck Centre for Extreme and Quantum Photonics, University of Ottawa, \\ Ottawa, Ontario K1N 6N5, Canada
}

(Received 6 January 2017; accepted 2 February 2017; published online 14 February 2017)

\begin{abstract}
We propose and perform an interference experiment involving a distributed angular double-slit and the orbital angular momentum (OAM) correlations of thermal light. In the experiment, two spatially separated angular apertures are placed in two correlated light beams generated by splitting the thermal light beam via a beam splitter. The superposition of the two spatially separated slits constitutes an angular double-slit in two-photon measurements. The angular interference pattern of the distributed double-slit is measured even though each beam interacts with a different part of the object. This scheme allows us to discriminate among different angular amplitude objects using a classical incoherent light source. This procedure has potential applications in remote sensing or optical metrology in the OAM domain. Published by AIP Publishing.

[http://dx.doi.org/10.1063/1.4976575]
\end{abstract}

It is established that apart from polarization and spin angular momentum, light also possesses an orbital angular momentum (OAM), ih per photon, arising from a helical phase structure of the beam. ${ }^{1-3}$ The associated OAM eigenstates form a complete, orthogonal, and infinite-dimensional basis, and have been demonstrated to be a useful degree of freedom for quantum information applications that require a high-dimensional Hilbert space. Furthermore, the OAM can also be used in imaging, optical metrology, and quantum information. ${ }^{4-6}$

Two-photon correlation has been studied in different contexts, such as ghost imaging, ghost diffraction, and distributed double-slit interference, which uses entangled twophoton state or incoherent thermal light source. ${ }^{7-12}$ So far, two-photon correlation has been theoretically proved and experimentally accessible. Several investigations have addressed this topic in regard to the high dimensional OAM structure. ${ }^{13,14}$ Ghost imaging and object identification explorations with OAM quantum correlations have been implemented. ${ }^{15-18}$ Experiments on angular ghost diffraction with entangled two-photon light source were reported, where angular double-slits were placed in the signal beam ${ }^{19}$ or with an angular double-slit in both signal and idler beams simultaneously. ${ }^{20}$ Optical vortices produced by the interference of random waves are intrinsic elements in a chaotic light. A special class of the optical vortices carries OAM, which is characterized by an azimuthal phase dependence of the form $e^{i l \phi}$. Here, $l$ is the OAM mode number and $\phi$ is the azimuthal angle..$^{21,22}$ Recently, Magaña-Loaiza et al..$^{23,24}$ have studied the nature of the correlations between different OAM components dependent on the phase of the fluctuations of

\footnotetext{
${ }^{\text {a) }}$ Author to whom correspondence should be addressed. Electronic mail: gaolu@cugb.edu.cn
}

pseudothermal light source. They showed that the thermal light correlation effects share similarities with the quantum correlations in the azimuthal degree of freedom, which has opened up a door to study thermal light correlations.

Although the double-slit interference with random optical wave fields has been discussed widely, ${ }^{25-28}$ none of the previous research works concerns a spatially distributed angular double-slit interference with the thermal light OAM components correlation measurement. Here, we explore a distributed double-slit interference phenomenon with thermal light infinite-dimensional OAM basis correlation. The thermal light beam is scattered by two spatially separated apertures to mimic the scattering by an angular double-slit, although none of the two apertures was an angular doubleslit. Rather, the superposition of the two apertures defines an angular double-slit, which determines the shape of the OAM second-order correlation spectrum. Two detectors are used to measure intensity correlations between two OAM components of a controllable incoherent light source. The first- and second-order interference patterns of the distributed angular double-slit are measured in far-field. Experimental results show that such correlations unveil the azimuthal structure of the source. An extension of the thermal light OAM correlation theory is shown to be able to describe our experimental results. Further numerical simulations prove that our experimental scheme can be used to discriminate amplitude angular object by thermal light with random phase fluctuations in the OAM domain, which liberates the requirement of the fragile quantum entanglement light source and is robust against environmental noise. The study here could provide new insights to further our understanding of correlation characteristics of an incoherent thermal light.

The experimental setup is shown in Fig. 1(a). The light from a helium neon laser is reflected from a digital micro 
a

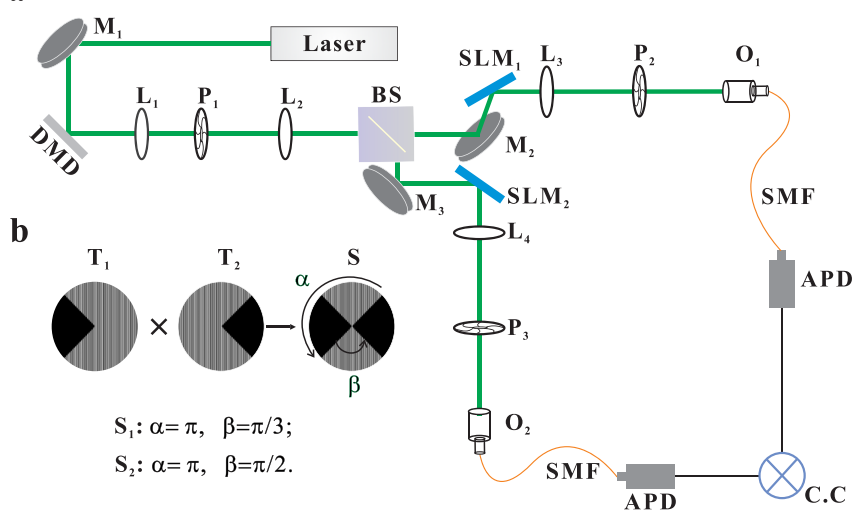

FIG. 1. (a) Schematic of the experimental setup. The laser beam is reflected by the DMD to generate the pseudo-thermal light. $L_{1}$ and $L_{2}$ are the two lenses to form a $4 \mathrm{f}$-optical system, and $P_{1}$ is a pinhole on the focal plane to isolate the first diffraction order of the structured beam. A series of random patterns are displayed on the DMD at a frequency of $1.4 \mathrm{kHz}$ to produce a random field of light. The generated beam is divided by a beam splitter to produce a test beam and a reference beam. The SLM in each arm is encoded by different OAM forked holograms and the detected object. (b) Distributed angular double-slit encoded with forked hologram. $S_{1}$ and $S_{2}$ are two distributed angular double-slits with different angular parameters.

device (DMD) to impose transverse fluctuations with thermal statistics onto the laser beam. A sequence (at a $1.4 \mathrm{kHz}$ writing rate) of random transverse structures possessing Kolmogorov statistics is impressed onto the beam to simulate thermal light, ${ }^{29}$ which has the similar effect as the rotating ground glass typically used to produce incoherent thermal light source. ${ }^{30}$ The divergence angle of the thermal light beam is less than $0.05 \mathrm{rad}$. Two identical copies of the beam are created by the non-polarizing beam splitter (BS), which are the reference beam and test beam. Each beam is sent to a spatial light modulator (SLM) onto which a computer-generated fork holograms and different transmission structures $T_{1}$ or $T_{2}$ are imposed, as shown in Fig. 1(b). Forked holograms corresponding to different OAM values are encoded to project out controllable OAM components. Then these beams are detected by avalanche photodetectors (APDs) and sent to a coincidence counting circuit (C.C). The objective $O_{1}$ ( or $O_{2}$ ) connected with APD in each beam is on the focal plane of the convex lens $L_{3}$ (or $L_{4}$ ), which collects the first-order Fourier spectrum of the beam reflected from the SLM carrying the OAM information. The measurement of thermal light OAM is carried out in the paraxial light. ${ }^{31}$ $M_{1}, M_{2}$, and $M_{3}$ are mirrors; $L_{1}$ and $L_{2}$ are the convex lenses which form a $4 \mathrm{f}$ optical system; $P_{1}$ is a pinhole acting as a spatial filter to isolate the first order of diffraction from the DMD; $P_{2}$ and $P_{3}$ are pinholes to filter first order of diffraction pattern from SLMs in each beam. SMF presents a single mode fiber.

In Fig. 1(b), the superposition of $T_{1}$ and $T_{2}$ is a single angular double-slit. In the experiment, we measured two different distributed angular double-slits $S_{1}$ and $S_{2}$. The angular slit width of $S_{1}$ and $S_{2}$ are $\pi / 3$ and $\pi / 2$, respectively. And the angular separation between the two angular slit centers $S_{1}$ and $S_{2}$ are both $\pi$.

The correlation measurement is carried out as the OAM value of the test beam is fixed with $l_{1}=0$ but $l_{2}$ scans in the reference beam from -7 to 7 . This task is performed by encoding the different forked holograms corresponding to certain OAM modes on the SLM in the reference beam.

The experimental results of the distributed angular double-slit $S_{1}$ are shown in Fig. 2. The normalized OAM intensity correlation between the two APDs is shown in Fig. 2(a), and the corresponding OAM second-order correlation function is $g^{(2)}\left(l_{1}, l_{2}\right)=\kappa\left\langle I_{1}\left(l_{1}\right) I_{2}\left(l_{2}\right)\right\rangle /\left\langle I_{1}\left(l_{1}\right)\right\rangle\left\langle I_{2}\left(l_{2}\right)\right\rangle$, where $\kappa$ is the detection efficiency. The OAM values corresponding to signal bars are $0, \pm 2$, and \pm 4 . Intensity profiles registered by the APD in the reference beam are shown in Fig. 2(b). The intensity profile has smooth distribution and does not exhibit any signal. It can be understood since the light source here is incoherent and generated by impressing random phase screens onto the DMD at high speed. The incoherent light source here obeys the Kolmogorov statistics distribution with the transverse coherence length $r_{0}=2 \mathrm{~mm}$.

Then, we carried out ghost angular Young's interference with the superposition of $T_{1}$ and $T_{2}$ in the test beam and light goes freely in the reference beam with no object. The correlation measurement is carried out as the OAM value in the test beam $l_{1}$ is fixed at 0 and in the reference beam $l_{2}$ scans from -7 to 7 . The normalized correlation measurement distribution profiles are shown in Fig. 2(c). In Fig. 2(d), it is the interference spectrum of the superposition of $T_{1}$ and $T_{2}$ illuminated by the coherent laser beam in the test beam. The spectrum in Fig. 2(c) is almost equivalent to that in Fig. 2(a), which proves that the distributed masks $T_{1}$ and $T_{2}$ can be joined through the incoherent light OAM correlation measurement.

The measurement processes of $S_{2}$ are the same as $S_{1}$. The experimental detection results are shown in Fig. 3. The signal bars here are corresponding to $l=0, \pm 2$, and \pm 6 which are different from $S_{1}$. It is because $S_{1}$ and $S_{2}$ have different angular parameters, which can be explained by the theoretical formula, Eq. (5), in the following paragraph.
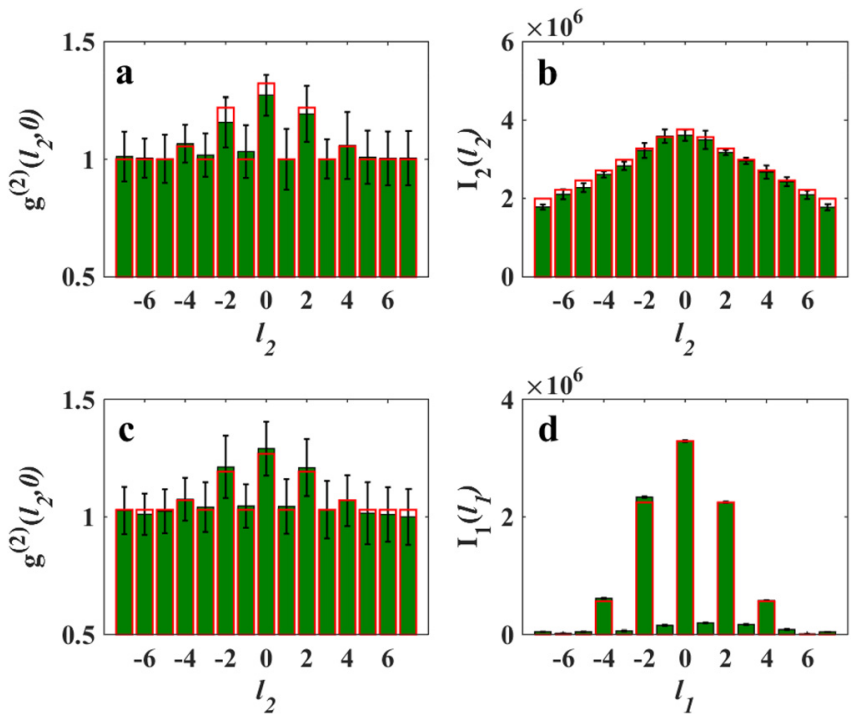

FIG. 2. Interference spectrum of distributed angular double-slit $S_{1}$ with thermal light OAM. (a) The normalized intensity correlation spectrum and (b) the intensity distribution spectrum; (c) the normalized intensity correlation spectrum of the superposition of $T_{1}$ and $T_{2}$ in the test beam and no object in the reference beam. (d) The angular double-slit interference spectrum of the superposition of $T_{1}$ and $T_{2}$ illuminated by coherent laser beam. Green solid bars are the experimental data and red line bars are the numerical simulations. 

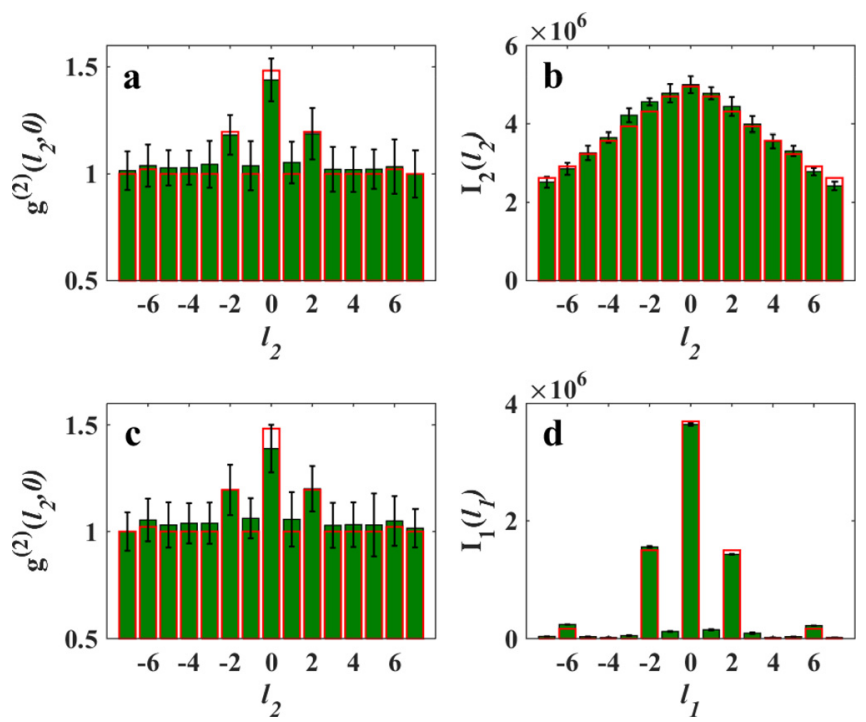

FIG. 3. Interference spectrum of distributed angular double-slit $S_{2}$ with thermal light OAM. (a) The normalized intensity correlation spectrum and (b) the intensity distribution spectrum; (c) the normalized intensity correlation spectrum of the superposition of $T_{1}$ and $T_{2}$ in the test beam and no object in the reference beam. (d) The angular double-slit interference spectrum of the superposition of $T_{1}$ and $T_{2}$ illuminated by coherent laser beam. Green solid bars are the experimental data and red line bars are the numerical simulations.

Even though there is no real angular double-slit in the experimental scheme, the far-field interference patterns of an angular double-slit structure are still achieved through the OAM correlation detection. The combination of masks $T_{1}$ and $T_{2}$ owes to the correlation characteristics of the azimuthal structure of the incoherent thermal light. The nature of the correlations between different OAM components of pseudo-thermal light comes from the strength of the fluctuations. ${ }^{23}$

The above experimental results can be explained by considering the OAM correlation properties of thermal light with the random phase fluctuation. When a thermal light beam is divided into two beams, the intensity correlation between them can be written as

$$
\begin{aligned}
G^{(2)}\left(l_{1}, l_{2}\right) & =\left\langle I_{1} I_{2}\right\rangle=\left\langle\psi_{l_{1}}^{*} \psi_{l_{2}}^{*} \psi_{l_{2}} \psi_{l_{1}}\right\rangle \\
& =\left\langle\psi_{l_{1}}^{*} \psi_{l_{1}}\right\rangle\left\langle\psi_{l_{2}}^{*} \psi_{l_{2}}\right\rangle+\left|\left\langle\psi_{l_{1}}^{*} \psi_{l_{2}}\right\rangle\right|^{2} \\
& =\left\langle I_{1}\right\rangle\left\langle I_{2}\right\rangle+\Delta G^{(2)}\left(l_{1}, l_{2}\right),
\end{aligned}
$$

where the optical field on the detection transverse plane is

$$
\psi_{l}=\int r d r d \phi(2 \pi)^{-1 / 2} \exp (-i l \phi) E(r) \Phi(r, \phi) T(\phi) .
$$

Here, $l$ is the OAM index, $E(r)$ represents the coherent optical field produced by the laser, $\Phi(r, \phi)$ is a particular realization of a DMD chaotic phase screen, and $T(\phi)$ describes the transmission function of the detected aperture.

The superposition of $T_{1}$ and $T_{2}$ forms an angular doubleslit function, and it can be written as

$$
S(\phi)=T_{1}(\phi) T_{2}(\phi)=\left\{\begin{array}{l}
1 \frac{\alpha-\beta}{2} \leq|\phi| \leq \frac{\alpha+\beta}{2} \\
0 \text { others, }
\end{array}\right.
$$

where $\alpha$ is the angular separation between the angular slit centers and $\beta$ is the angular width.

Substituting Eqs. (2) and (3) into Eq. (1), we can calculate the intensity and the second-order OAM fluctuation correlation functions as

$$
\begin{gathered}
\left\langle I_{j}\right\rangle=\frac{1}{2 \pi} \int r_{j} d r_{j}\left|E\left(r_{j}\right)\right|^{2} d \phi_{j} T_{j}\left(\phi_{j}\right) \propto \text { const }, j=1,2, \\
\Delta G^{(2)}\left(l_{1}, l_{2}\right) \\
=\frac{1}{4 \pi^{2}} \times\left.\left.\left|\int r_{1} d r_{1} d \phi_{1}\right| E\left(r_{1}\right)\right|^{2} S\left(\phi_{1}\right) \exp \left[i \phi_{1}\left(l_{1}-l_{2}\right)\right]\right|^{2},
\end{gathered}
$$

where fully incoherent thermal light is considered, and the light field correlation function is $\left\langle\Phi^{*}\left(r_{1}, \phi_{1}\right) \Phi\left(r_{1}^{\prime}, \phi_{1}^{\prime}\right)\right\rangle$ $=\left(1 / r_{1}\right) \delta\left(r_{1}-r_{1}^{\prime}\right) \delta\left(\phi_{1}-\phi_{1}^{\prime}\right)$. By submitting Eq. (3) into Eq. (5), we could obtain the second order OAM correlation item as $\Delta G^{(2)}\left(l_{1}, l_{2}\right)=C \beta^{2} \sin c^{2}\left(\frac{\beta\left(l_{1}-l_{2}\right)}{2}\right) \cos ^{2}\left(\frac{\alpha\left(l_{1}-l_{2}\right)}{2}\right)$, which is a symmetrical function with $l_{1}$ and $l_{2}$. The numerical results with this theoretical analysis are in good agreement with the experimental data.

By making use of the correlated characteristics of the above experimental scheme, we proposed a thermal light OAM correlation object discrimination scenario and carried out the numerical simulations. The simulation results are shown in Fig. 4. This scenario is based on the experimental scheme shown in Fig. 1. $T_{1}$ in the test beam is set as the target object to be discriminated. $T_{2}$ is the tentative object in the reference beam, whose angular width or angular orientation could change during the process of discrimination. First, we carry out ghost angular Young's interference measurement, where the beam in the reference beam goes freely with no tentative object. The correlated OAM spectrum is shown in Fig. 4(a), which is taken as the comparison criterion spectrum of the measurement. Then, $T_{2}$ is inserted in the reference beam, whose angular width or orientation changes during the process of discrimination. The OAM correlation measurement results are shown in Figs. 4(b)-4(d). Only if the chosen $T_{2}$ has the same angular distribution as the target object $T_{1}$, we could achieve the spectrum shown in Fig. 4(b) which has the same distribution as in Fig. 4(a). Otherwise, if the chosen $T_{2}$ has a different angular structure with the target object $T_{1}$, we could just achieve different spectra from that in Fig. 4(a), which are shown in Figs. 4(c) and 4(d). So when we obtain the same spectrum as that in Fig. 4(a), we could state that the target object $T_{1}$ has the same angular distribution as $T_{2}$. The detected target object $T_{1}$ has been discriminated.

The above results can be understood by Eqs. (3) and (5). For the amplitude transmission object with angular distribution, if $T_{2}=T_{1}$ the correlation effect will lead to $T_{1} T_{2}$ $=\left|T_{1}\right|^{2}=T_{1}$. But this cannot happen with $T_{2} \neq T_{1}$. So in Figs. 4(a) and 4(b), the spectra have the same distributions with each other, but Figs. 4(c) and 4(d) have different spectra from Fig. 4(a). This scenario can provide a new thinking to discriminate the object with angular distribution by making use of the OAM correlation with thermal light. It may have potential applications in remote sensing and optical metrology in the OAM-domain with the classical incoherent light source. 

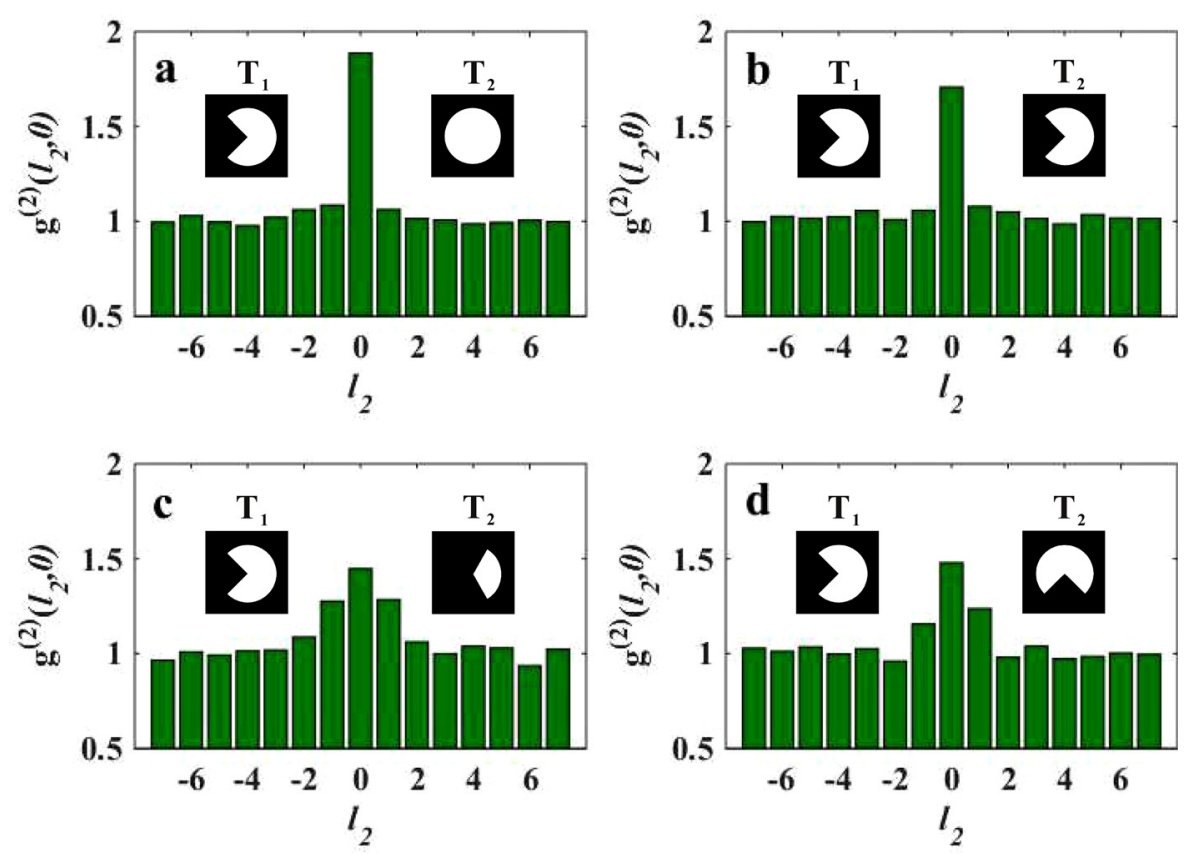

FIG. 4. Numerical simulation results of object discrimination in the OAM domain. (a) The correlated OAM spectra with the reference beam going freely. (b) The similar OAM spectra to (a) when $T_{2}$ has the same angular structure with $T_{1}$; (c) and (d) different OAM spectra from (a) when $T_{2}$ has different angular structure with $T_{1}$. The numerical simulated results are achieved from 2000 frames statistical average.
In summary, we have investigated a subtle aspect of the Hanbury Brown and Twiss (HBT) effect related with thermal light azimuthal freedom. Our experimental results show that the interference patterns of a distributed angular double-slit can be measured by intensity correlations in the OAM components of the pseudothermal light source. It is possible to achieve the detected object's OAM interference patterns not only when the illuminating beam passes through a single object $^{23}$ but also when the separated beams pass through different parts of the distributed object. Different from the former research works, ${ }^{17,27}$ we extend the distributed Young's double-slit interference correlation measurement to the OAM domain even with the incoherent thermal light source. It proves that quantum entanglement is not necessary for the distributed angular double-slit interference in the OAM domain. Further numerical simulations prove that our experiment scenario could be used to discriminate transmission amplitude object with angular distribution, which has potential applications in optical OAM-based metrology and remote sensing with the classical incoherent light source when the fragile quantum entangled state is not available.

This work was supported by the National Natural Science Foundation of China (Grant No. 11504337), the Funding of China Scholarship Council (Grant No. 1508130007), and the Fundamental Research Funds for the Central Universities (No. 53200859334).

${ }^{1}$ L. Allen, M. W. Beijersbergen, R. J. C. Spreeuw, and J. P. Woerdman, Phys. Rev. A 45, 8185 (1992).

${ }^{2}$ A. Mair, A. Vaziri, G. Weihs, and A. Zeilinger, Nature 412, 313 (2001).

${ }^{3}$ M. J. Padgett, J. Courtial, and L. Allen, Phys. Today 57(5), 35 (2004).

${ }^{4}$ L. Torner, J. P. Torres, and S. Carrasco, Opt Express 13, 873 (2005).

${ }^{5}$ O. S. Magaña-Loaiza, M. Mirhosseini, B. Rodenburg, and R. W. Boyd, Phys. Rev. Lett. 112, 200401 (2014).

${ }^{6}$ N. Bozinovic, Y. Yue, Y. Ren, M. Tur, P. Kristensen, H. Huang, A. E. Willner, and S. Ramachandran, Science 340, 1545 (2013).
${ }^{7}$ R. S. Bennink, S. J. Bentley, and R. W. Boyd, Phys. Rev. Lett. 89, 113601 (2002).

${ }^{8}$ R. S. Bennink, S. J. Bentley, and R. W. Boyd, Phys. Rev. Lett. 92, 033601 (2004).

${ }^{9}$ J. Cheng and S. Han, Phys. Rev. Lett. 92, 093903 (2004).

${ }^{10}$ K. Wang and D. Z. Cao, Phys. Rev. A 70, 041801(R) (2004).

${ }^{11}$ Y. Cai and S. Y. Zhu, Opt. Lett. 29, 2716 (2004).

${ }^{12}$ A. Gatti, E. Brambilla, M. Bache, and L. A. Lugiato, Phys. Rev. Lett. 93, 093602 (2004).

${ }^{13}$ S. Franke-Arnold, S. M. Barnett, M. J. Padgett, and L. Allen, Phys. Rev. A 65, 033823 (2002).

${ }^{14}$ J. P. Torres, A. Alexandrescu, and L. Torner, Phys. Rev. A 68, 050301 (2003).

${ }^{15}$ N. Uribe-Patarroyo, A. Fraine, D. S. Simon, O. Minaeva, and A. V. Sergienko, Phys. Rev. Lett. 110, 043601 (2013).

${ }^{16}$ B. Jack, J. Leach, J. Romero, S. Franke-Arnold, M. Ritsch-Marte, S. M. Barnett, and M. J. Padgett, Phys. Rev. Lett. 103, 083602 (2009).

${ }^{17}$ L. Chen, J. Leach, B. Jack, M. J. Padgett, S. Franke-Arnold, and W. She, Phys. Rev. A 82, 033822 (2010).

${ }^{18}$ L. Chen, J. Lei, and J. Romero, Light: Sci. Appl. 3, e153 (2014).

${ }^{19}$ A. K. Jha, B. Jack, E. Yao, J. Leach, R. W. Boyd, G. S. Buller, S. M. Barnett, S. Franke-Arnold, and M. J. Padgett, Phys. Rev. A 78, 043810 (2008).

${ }^{20}$ A. K. Jha, J. Leach, B. Jack, S. Franke-Arnold, S. M. Barnett, R. W. Boyd, and M. J. Padgett, Phys. Rev. Lett. 104, 010501 (2010).

${ }^{21}$ M. V. Berry, J. Phys. A 11, 27-37 (1978).

${ }^{22}$ M. R. Dennis, K. OHolleran, and M. J. Padgett, Prog. Opt. 53, 293-363 (2009).

${ }^{23}$ O. S. Magaña-Loaiza, M. Mirhosseini, R. M. Cross, S. M. H. Rafsanjani, and R. W. Boyd, Sci. Adv. 2, e1501143 (2016).

${ }^{24}$ Z. Yang, O. S. Magaña-Loaiza, M. Mirhosseini, Y. Zhou, B. Gao, L. Gao, S. M. Hashemi Rafsanjani, G. Long, and R. W. Boyd, e-print arXiv:1609.08741.

${ }^{25}$ J. Xiong, D. Cao, F. Huang, H. G. Li, X. J. Sun, and K. Wang, Phys. Rev. Lett. 94, 173601 (2005).

${ }^{26}$ E. J. S. Fonseca, P. H. S. Ribeiro, S. Padua, and C. H. Monken, Phys. Rev. A 60, 1530 (1999).

${ }^{27}$ L. Gao, J. Xiong, L. Lin, W. Wang, S. Zhang, and K. Wang, Opt. Commun. 281, 2838 (2008).

${ }^{28}$ I. Vidal, D. P. Caetano, E. J. S. Fonseca, and J. M. Hickmann, Europhys. Lett. Assoc. 82, 34004 (2008).

${ }^{29}$ M. Mirhosseini, O. S. Magaña-Loaiza, C. Chen, B. Rodenburg, M. Malik, and R. W. Boyd, Opt. Express 21, 30196 (2013).

${ }^{30}$ F. T. Arecchi, Phys. Rev. Lett. 15, 912 (1965).

${ }^{31}$ J. W. Goodman, Introduction to Fourier Optics, 2nd ed. (Stanford University, 1996). 